\title{
Forestación peridoméstica en Patagonia y conocimiento ecológico tradicional: un estudio de caso
}

\author{
M. Betina Cardoso \& Ana Haydeé Ladio*
}

Instituto de Investigaciones en Biodiversidad y Medio Ambiente (INIBIOMA-CONICET), Universidad Nacional del Comahue, Quintral 1250, San Carlos de Bariloche, Río Negro, Argentina.

\begin{abstract}
Resumen - La estepa patagónica constituye un paisaje cultural que es recreado permanentemente por la acción humana. En este estudio de caso, se analizó la forestación peri-domiciliaria con especies arbóreas en una pequeña comunidad incluyendo la riqueza de plantas que son utilizadas y las motivaciones asociadas a su uso. En la población Mapuche de Pilkiniyeu del Limay ("Cañadón de las ardillas") en la provincia de Río Negro, se realizaron entrevistas semi-estructuras y libres a 28 informantes. Los datos fueron analizados con métodos uni y multivariados. Los principales resultados indican que se utilizan nueve especies de origen exótico. Dichas forestaciones constituyen un espacio vital de alto carácter social dado que es un lugar de reuniones y de esparcimiento. Las principales motivaciones para la forestación entre los habitantes es la de tener materiales para la construcción de cercos (96\%), el reparo del viento y el frío (86\%), el uso leñatero (43\%) y el forraje para el ganado (11\%). Asimismo, el MSD mostró un sistema compartido de valoraciones acerca de la forestación. El conocimiento ecológico tradicional que utilizan los pobladores a la hora de usar y seleccionar especies combustibles no parece influir en las motivaciones para forestar en sus casas. Este hecho podría ser interpretado como que la forestación es una práctica generalista que está fuertemente arraigada e influenciada por la transmisión oblicua llevada a cabo por técnicos externos de promoción agropecuaria que visitan y asisten a esta comunidad.
\end{abstract}

Palabras claves: estepa, Mapuche, percepción cultural, plantas leñosas.

\begin{abstract}
Peridomestic forestation in Patagonia and traditional ecological knowledge: a case study) - The Patagonian steppe is a cultural landscape which is constantly being recreated by man. In this case study, peridomestic forestation of tree species was analysed in a small community, including the richness of plants used and the reasons behind their choice. In the Mapuche population of Pilkiniyeu del Limay (Río Negro) free and semi-structured interviews were carried out with 28 interviewees. The data was analysed using both uni- and multivariate methods. Results indicate that nine exotic species are used. These forested areas, used for meetings and recreation, provide useful living space and are of great social importance. The principal motivation behind this forestation is the need for materials for building fences (96\%), to provide shelter from the elements $(86 \%)$, firewood $(43 \%)$, and cattle fodder $(11 \%)$. The MSD revealed a shared value system with respect to the forestation. The traditional ecological knowledge that people use in the selection and use of firewood species does not seem to have an influence on choices made when planting trees around their homes. This could indicate that forestation is a general practice deeply rooted and influenced by the oblique transmission carried out by external agriculture agents who visit and offer help to this community.
\end{abstract}

Aditional key words: cultural perception, Mapuche, steppe, woody plants.

Desde una mirada etnobiológica, el paisaje constituye un escenario dinámico que refleja una intrincada red de personas, conocimientos, lugares y recursos. Estos escenarios son producto de prácticas humanas (tradicionales y/o foráneas) que reflejan valores (sociales, espirituales y ecológicos) y que varían con la percepción temporal y espacial de cada grupo sociocultural en un entorno determinado (Davidson-Hunt \& Berkes 2003; Berkes \& Davidson-Hunt 2006).

En la meseta patagónica de Río Negro, la mayor parte de la población corresponde a campesinos rurales, minifundistas, que viven de la cría del ganado y sobreviven ajustándose a situaciones de marcadas limitaciones socioambientales (Menni 1999; Peralta 2002; ICEPH 2010). El paisaje estepario, si bien representa un ambiente hostil

\footnotetext{
*Autora de correspondencia: aladio2002@yahoo.com.ar Editor responsável: Rômulo Romeu Nobrega Alves Recibido: 12 mayo 2011; acepto: 11 ago. 2011.
}

para las comunidades humanas, brinda recursos que son utilizados por los pobladores desde tiempos ancestrales tanto para suplir sus necesidades materiales como espirituales (Ladio 2006, 2011a; Ladio \& Lozada 2009). Asimismo, la estepa constituye un paisaje cultural que es recreado permanentemente por la acción humana. Por ejemplo, ha sido extensamente documentado cómo las prácticas de sobrepastoreo han alterado los sistemas ecológicos en Patagonia (Raffaele 1996, 1999; Raffaele \& Veblen 2001; Peralta 2002; Paruelo et al. 2006; Rush et al. 2008). Sin embargo, poco sabemos sobre el efecto de otras intervenciones humanas que generan cambios de paisaje como es el caso de la forestación peridoméstica.

Estudios recientes dan cuenta que las poblaciones de la estepa patagónica dependen mayormente de la recolección de plantas leñosas combustibles para cubrir sus necesidades de calor (Cardoso et al. 2010) y, en menor medida, de especies medicinales y comestibles que son extraídas directamente de áreas naturales aledañas (Ladio 
\& Lozada 2009; Molares \& Ladio 2009; Ladio 2011b). Frente a la rigurosidad climática y la escasez del recurso leñero, el uso de biocombustibles es complementado por la recolección de estiércol y por la compra de leña proveniente de otras regiones del país (Cardoso et al. 2010). Por otro lado, distintos actores sociales y organismos gubernamentales de la región han detectado la falta de leña como una problemática urgente, y han iniciado distintos planes de forestación en la región (Peralta 2002; Lebed 2003; Izquierdo et al. 2009).

Sin embargo, poco se ha tenido en cuenta la percepción local sobre las motivaciones y preferencias de las personas asociadas al uso del paisaje y su transformación. Distintas aproximaciones al estudio del conocimiento ecológico tradicional dan cuenta que una mayor diversidad de conocimientos, por ejemplo, sobre plantas, actúa directamente sobre la percepción y la acción de los habitantes sobre su ambiente (Berkes \& DavidsonHunt 2006; Ladio 2011b).

Asimismo, desde una aproximación cognitiva se ha establecido que una mayor experiencia y adquisición de saberes genera en los individuos un cambio gradual en el proceso de aprendizaje y apropiación de la naturaleza (Nolan 2001; Davidson-Hunt \& Berkes 2003). Por ejemplo, la acción de recolectar asiduamente plantas leñosas para uso combustible permitiría a los individuos evaluar aspectos ligados a percibir amenazas o cambios en la disponibilidad, y tomar decisiones sobre cómo mantener o propiciar mayores recursos leñosos. Dichas decisiones son tomadas desde la propia percepción cultural, bajo conceptos y valores particulares que reflejan la historia y la apropiación del paisaje.

Desde la colonización del territorio por parte del Estado argentino, la forestación en la Patagonia se encuentra vinculada a grandes extensiones de especies exóticas maderables como por ejemplo las pináceas: Pinus ponderosa Douglas ex Lawson \& C. Lawson y/o Pseudotsuga menziesii (Mirb.) Franco para su explotación comercial a gran escala (Peralta 2002; Broquen et al. 2003; Sarasola et al. 2006; Nuñez \& Raffaele 2007; Rush et al. 2008; Orellana \& Raffaele 2010). En cambio, la forestación doméstica es una práctica de menor escala en el ámbito domiciliario que se ha desarrollado en la estepa como una alternativa para combatir, en primera medida, la acción del viento y obtener abrigo frente a las bajas temperaturas.

En este trabajo, en una comunidad pequeña y aislada de la estepa patagónica estudiaremos aspectos ligados a la percepción cultural sobre la forestación doméstica y su relación con el conocimiento ecológico tradicional. Nuestras principales preguntas son: $1-¿$ Cuál es la importancia cultural de la forestación domiciliaria? ¿Qué especies incluye? 2¿Cuáles son las motivaciones de los pobladores para forestar? ¿Están asociadas unas con otras? 3- ¿El mayor conocimiento sobre plantas leñosas combustibles de la región influye en las distintas motivaciones de las personas para forestar?

Área de estudio. Pilkiniyeu del Limay $\left(41^{\circ} 02^{\prime} \mathrm{S}\right.$ y $70^{\circ} 51^{\prime} \mathrm{W}$ ) está emplazada en un paisaje de estepa con arbustos bajos y gramíneas, y con un relieve formado por valles y mallines esteparios, perteneciente a la región fitogeográfica Provincia del Monte (Cabrera \& Willink 1973). El estado actual de desertificación puede considerarse de medio-leve a medio-grave (Bran et al. 2000). El clima es predominantemente árido y frío, con precipitaciones entre 150 a $300 \mathrm{~mm}$ anuales, concentradas en otoño e invierno en forma de lluvia o nieve, y la temperatura media anual es de 8 a $10^{\circ} \mathrm{C}$ (Bran et al. 2000).

Dicha población rural Mapuche se encuentra conformada por 55 familias, siendo el pueblo más cercano Comallo (2000 habitantes) ubicado a $120 \mathrm{~km}$ y la ciudad más cercana San Carlos de Bariloche (130.000 habitantes) a 220 $\mathrm{km}$. Esta población vive en este sitio desde 1992 dado que la misma fue relocalizada por la construcción de la Central Hidroeléctrica Piedra del Águila (Radovich \& Balazote 1991), aunque anteriormente habitaba un ambiente cercano, pero con mayores aptitudes ecológicas. La principal actividad económica es la cría de ganado ovino y caprino y la confección de artesanías en el marco de una economía familiar de subsistencia. En casi todos los hogares se practica el cultivo de la tierra realizando huertos domiciliarios. Por otra parte, la comunidad no posee red de electricidad, sólo el $30 \%$ accede al servicio de manera muy restringida y alimentada con gasoil. El $80 \%$ de las personas poseen ascendencia Mapuche, sin embargo sólo el 20\% son bilingües y hablan el "Mapuzungun". Por otro lado, el $30 \%$ de los informantes completaron sus estudios primarios. En la actualidad todos los niños asisten a una escuelahogar ubicada en el mismo paraje.

\section{Materiales y Métodos}

La autoridad máxima de la comunidad, "el lonko", expresó su conformidad para que se realizara la investigación, y posteriormente se obtuvo el consentimiento de cada participante (Alexiades 1996; Albuquerque et al. 2010). Debido a la larga distancia entre los hogares de la comunidad, el criterio de selección de los informantes se basó en visitas al azar de todos aquellos hogares a los cuales se pueda acceder. Finalmente, durante enero y marzo de 2009 , un total de 28 familias fueron entrevistadas (60\% de la población) en sus hogares en el idioma español. Todos los informantes resultaron adultos de diferentes edades (media $=36$, mín. 24 y máx. 76), 16 mujeres y 12 hombres.

La metodología etnográfica incluyó observación participante y entrevistas abiertas para la pregunta 1. En el caso de las preguntas siguientes se realizaron entrevistas semi-estructuradas y enlistados libres (Alexiades 1996; Albuquerque et al. 2010) enfocándonos en las motivaciones para forestar con especies leñosas en el peridomicilio. Se 
estableció como peridomicilio al ámbito que rodea la casa, el jardín y la huerta que en general corresponde a un área de aproximadamente $1000-1500 \mathrm{~m}^{2}$ y puede incluir o no corrales y galpones. Por otra parte, la riqueza de especies leñosas combustibles citadas por los informantes de esta comunidad fue tomada de Cardoso et al. (2010). Se colectaron muestras de ramas y troncos de especies usadas para forestar por los pobladores confeccionándose un herbario de referencia que fue depositado en el Laboratorio Ecotono (UNCo). La filiación taxonómica sigue Correa (1969-1998) y Ezcurra \& Brion (2005).

Análisis de datos. Las frecuencias de citas fueron analizadas cuali-cuantitativamente con estadística no paramétrica y multivariada considerando la naturaleza categórica de los datos (Agresti 1996). La frecuencia de forestación fue calculada teniendo en cuenta el número de informantes que citó cada especie sobre el número total de informantes (Tabla 1). Las respuestas de los informantes sobre las motivaciones para forestar fueron asignadas a categorías éticas: 1- reparo: cultivo de especies leñosas con el objetivo de generar reparo contra el viento, el frío y propiciar sombra; 2- leña: cultivo de especies leñosas con fines combustibles domiciliarios; 3- forraje: cultivo de especies leñosas como suplemento alimentario para el ganado; y 4- construcción: cultivo de especies leñosas para construcción de cercos, corrales, techos, y muebles. Como medida de un mayor o menor conocimiento ecológico tradicional sobre plantas combustibles silvestres, se clasificó a los informantes en dos categorías en función de la media de especies citadas por persona $(6,1 \pm 2,59$ especies $)$, a saber: 1 - poco (0-6 especies/persona) y 2- mucho (+ de 6 especies/persona). Esta categorización refleja un mayor o menor acercamiento de los informantes al uso de los recursos leñosos, datos que no mostraron variación en función de la edad ni del género (Cardoso et al. 2010). La frecuencia de citas de ambos grupos fue analizada con test binomiales y test de Fisher Exact Test ( $\mathrm{p}<0,05)$. Asimismo, se analizó la asociación del número de especies silvestres combustibles y la suma de las distintas motivaciones asignadas por persona con la correlación de Spearman (Agresti 1996).

Con el objeto de obtener una visión integral del comportamiento de las respuestas acerca de las motivaciones para forestar, se empleó el MDS (Análisis de escalamiento multidimensional). El MDS provee un arreglo espacial de los datos que muestra sus posiciones relativas tomando como base la matriz de datos de respuestas positivas y negativas de cada informante de modo de poder identificar patrones de acuerdo y similitud (Hair et al. 1998). La proporción de la variación que es explicada por este arreglo se midió con el valor del $\mathrm{R}^{2}$ (varía entre 0 y 1 ), y con el stress que estima el grado de ajuste de las distancias creadas cuyos valores de buen ajuste deben ser inferiores a 0,1 (Hair et al. 1998).

\section{Resultados y Discusión}

Importancia cultural de la forestación domiciliaria en Pilkiniyeu del Limay. En Pilkiniyeu, todas las familias forestan alrededor de sus casas para fines diversos. Se registraron nueve especies en total, siendo las más importantes las especies de los géneros Salix L. (sauces) y Populus L. (álamos) (Figura 1; Tabla 1). Estas especies son plantas exóticas proporcionadas por técnicos de INTA (Instituto Nacional de Tecnología Agropecuaria) que concurren a estos parajes y son distribuidas entre los pobladores por los propios vecinos. Las plantas leñosas de mayor consenso se distinguen por ser especies de crecimiento rápido en ambientes adversos (ej. álamos), sin embargo las especies con menor consenso se caracterizan por ser poco resistentes al frío (ej. sauce llorón y sauce eléctrico; Tabla 1) (Barreiro 2007; Steinbach et al. 2007; Rush et al. 2008), mostrando que la selección de especies responde a una práctica acertada.

Dado que la comunidad se encuentra en una zona árida con vientos fuertes y escasez de agua, los árboles cultivados por los habitantes son cuidadosamente protegidos del ganado y logran un buen desarrollo en pocos años. Llamativamente en esta comunidad, no se realiza forestación con plantas nativas. Los técnicos en la zona han promovido el cultivo de especies exóticas entre los habitantes, dado su rápido crecimiento, fisiológicamente adaptado, con respecto a las especies nativas (Moles et al. 2008). Asimismo, estas especies en su mayoría son caducas y de madera blanda por lo que su dosel en otoño e invierno pierde parte de su capacidad de protección térmica (Tabla 1). Por otro lado, los pobladores perciben y comentan las apreciadas cualidades de las maderas nativas, sin embargo destacan su lento desarrollo.

\section{Frecuencia de forestación de especies leñosas en Pilkiniyeu del Limay}

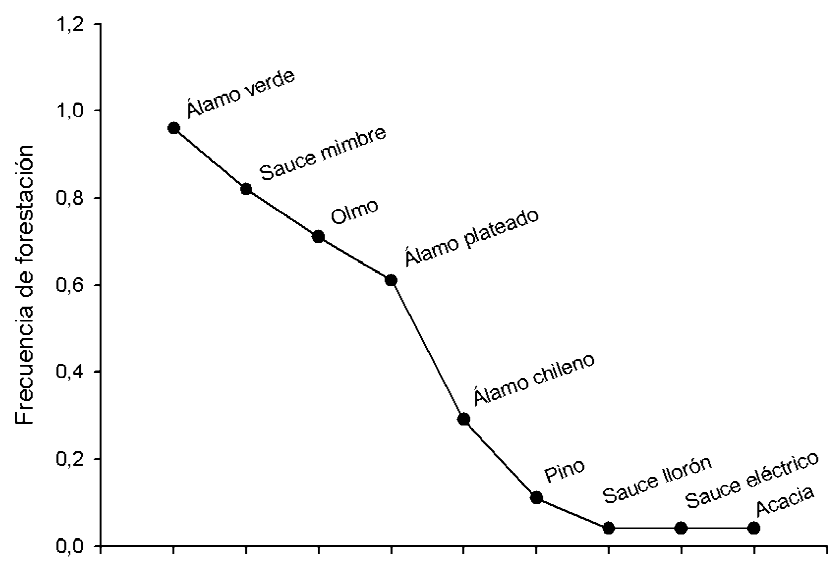

Figura 1. Principales especies forestadas en Pilkiniyeu del Limay (Río Negro, Argentina). La frecuencia de forestación es el número de citas de cada especie en función del total de informantes.

Sitientibus série Ciências Biológicas 11(2): 321-327. 2011. 
Tabla 1. Árboles utilizados para forestación peridoméstica en Pilkiniyeu del Limay (Río Negro, Argentina). La Frecuencia de cita es el número de citas en relación al total de entrevistados $(\mathrm{N}=28)$. Otros usos corresponden a otras formas de utilización según Valdora \& Soria (1999), Steinbach et al. (2007), Barreiro (2007), Alvarez et al. (2009).

\begin{tabular}{|c|c|c|c|c|c|c|c|}
\hline Nombre científico & $\begin{array}{l}\text { Nombre } \\
\text { vernacular }\end{array}$ & $\begin{array}{l}\text { Familia } \\
\text { botánica }\end{array}$ & $\begin{array}{l}\text { Origen } \\
\text { biogeográfico }\end{array}$ & $\begin{array}{l}\text { Frecuencia } \\
\text { de cita }\end{array}$ & Follaje & Madera & Otros usos \\
\hline Populus nigra L. & $\begin{array}{l}\text { Álamo } \\
\text { verde }\end{array}$ & Salicaceae & $\begin{array}{l}\text { Eurasia y Norte de } \\
\text { África }\end{array}$ & 89 & Caduco & Blanda & $\begin{array}{l}\text { Ornamental, } \\
\text { medicinal, } \\
\text { cajonería y } \\
\text { celulosa, } \\
\text { fabricación de tallas } \\
\text { y suecos }\end{array}$ \\
\hline Salix viminalis L. & $\begin{array}{l}\text { Sauce } \\
\text { mimbre }\end{array}$ & Salicaceae & Eurasia & 79 & Perenne & Blanda & $\begin{array}{l}\text { Contención de } \\
\text { riberas, cestería }\end{array}$ \\
\hline Ulmus minor Mill. & Olmo & Ulmaceae & Eurasia & 71 & Caduco & Dura & $\begin{array}{l}\text { Ornamental, } \\
\text { revestimientos }\end{array}$ \\
\hline Populus alba $\mathrm{L}$. & $\begin{array}{l}\text { Álamo } \\
\text { plateado }\end{array}$ & Salicaceae & $\begin{array}{l}\text { Eurasia y Norte } \\
\text { de África }\end{array}$ & 61 & Caduco & Blanda & $\begin{array}{l}\text { Ornamental, } \\
\text { medicinal, } \\
\text { cajonería, celulosa, } \\
\text { fabricación de tallas } \\
\text { y suecos }\end{array}$ \\
\hline $\begin{array}{l}\text { Populus } \times \text { canadensis } \\
\text { Moench. }\end{array}$ & $\begin{array}{l}\text { Álamo } \\
\text { chileno }\end{array}$ & Salicaceae & Canada & 29 & Caduco & Blanda & Cajonería, celulosa \\
\hline $\begin{array}{l}\text { Pinus contorta } \\
\text { Douglas \& Loudon }\end{array}$ & Pino & Pinaceae & América del Norte & 18 & Perenne & Blanda & Forestal \\
\hline $\begin{array}{l}\text { Robinia pseudo-acacia } \\
\text { L. }\end{array}$ & Acacia & Fabaceae & Estados Unidos & 3.6 & Caduco & Dura & $\begin{array}{l}\text { Oramental, control } \\
\text { de erosión, } \\
\text { melífera, tóxica, } \\
\text { carpintería, } \\
\text { revestimientos }\end{array}$ \\
\hline $\begin{array}{l}\text { Salix erithroflexuosa } \\
\text { Thunb. }\end{array}$ & $\begin{array}{l}\text { Sauce } \\
\text { eléctrico }\end{array}$ & Salicaceae & China y Corea & 3.6 & Caduco & Blanda & $\begin{array}{l}\text { Ornamental, } \\
\text { contención de } \\
\text { riberas }\end{array}$ \\
\hline Salix babylonica L. & Sauce llorón & Salicaceae & China & 3.6 & Caduco & Blanda & Ornamental, mítico \\
\hline
\end{tabular}

El uso de "alamedas" en los peri-domicilios patagónicos ha sido extensamente documentado en las zonas rurales de la Argentina (Lebed 2003; Barreiro 2007; Izquierdo et al. 2009). Desde el punto de vista ambiental, las forestaciones domiciliarias son adaptativas porque constituyen un sistema de mitigación de temperatura, la lluvia o nieve y de los vientos. Según Puntieri \& Grosfeld (2009), el tamaño y densidad del follaje, determina el grado de amortiguación de los fenómenos físicos ambientales, y por ende las forestaciones peridomésticas de Pilkiniyeu constituyen un buen sistema de amortiguamiento.

Adicionalmente, desde el punto de vista cultural, las forestaciones domiciliarias constituyen un espacio vital, delimitando la estructura hogareña y protegiendo la casa. Los pobladores lo distinguen como un ambiente agradable y reparado para compartir momentos en familia, parientes o visitas, hecho que le otorga un carácter social significativo. Cabe señalar que aldeas como la de Pilkiniyeu donde la distancia entre casas supera los $10 \mathrm{~km}$, el conjunto de copas cumple una función insigne "o de marca" de asentamiento humano, tanto del presente como del pasado que puede ser visualizado a gran distancia y cuyo rol en las comunicaciones y las interacciones sociales no puede ser menospreciado, por lo que aparecen según los entrevistados como referencia de caminos, y suelen ser un punto estratégico de encuentro.

Las especies cultivadas en Pilkiniyeu también son registradas en la bibliografía con otros usos secundarios, distinguiéndose el control de la erosión por vientos y lluvia, la fabricación de utensilios de la vida cotidiana, y el uso ornamental (Tabla 1). Se ha documentado que al sauce llorón se le atribuye uso mítico, en general asociado a la tristeza y la melancolía (Barreiro 2007; Steinbach et al. 2007), sin embargo este aspecto no fue registrado en Pilkiniyeu.

Percepciones y motivaciones de los pobladores para forestar en la estepa. El $100 \%$ de los entrevistados realiza cultivo de árboles en sus predios, dicha tarea es realizada por mujeres y hombres. Sin embargo, las mujeres se encargan de la forestación cercana al hogar, mientras que los hombres se dedican a plantaciones peri-domiciliarias mayores que abarcan un espacio más amplio, como es el caso de alamedas.

Las principales motivaciones para forestar en la población de Pilkiniyeu del Limay están relacionadas con el aprovisionamiento de materiales leñosos para la construcción de corrales y cercos $(96 \%$ de los informantes, test Binomial, $\mathrm{p}<0,05)$. En orden de importancia le sigue la utilización para reparo, citado por el $86 \%$ de la población (test Binomial, $\mathrm{p}<0,05)$, el uso leñatero $(43 \%$, test Binomial, $\mathrm{p}=0,571)$ y el cultivo de especies leñosas con propósitos de forraje $(11 \%$, test Binomial, $\mathrm{p}<0,05)$. Los informantes 
nombraron directamente argumentos vinculados al embellecimiento del lugar con especies arbóreas salvo en un solo caso. Por otra parte, es llamativo que cerca de la mitad de las personas no nombrara el uso leñatero como principal razón para la forestación, a pesar de los problemas generales de aprovisionamiento de combustible. Este fenómeno podría estar relacionado con el hecho de que la comunidad extrae preferentemente leña del matorral nativo como sucede en otras comunidades del país (Rodríguez y López 2006; Cardoso et al. 2010).

Cabe señalar que en las ciudades, por ejemplo distintas especies de Populus son utilizadas como una barrera visual y sonora que modera el ruido de las autopistas (Barreiro 2007; Puntieri \& Grosfeld 2009). Estos conceptos no fueron encontrados entre los informantes de Pilkiniyeu, reflejando cómo las mismas plantas pueden ser forestadas por razones absolutamente diferentes entre los distintos grupos humanos. En concordancia con lo encontrado por Jagger \& Luckert (2008), las forestaciones domiciliarias en Pilkiniyeu constituyen una estrategia ligada a contrarrestar la vulnerabilidad social y ambiental.

Conocimiento botánico tradicional sobre plantas leñosas combustibles y motivaciones hacia la forestación. No se encontró una asociación positiva entre el número de plantas leñosas silvestres recolectadas por persona como combustible y una mayor cantidad de razones argumentadas para forestar en el área $(\mathrm{p}=0,839, \mathrm{n}=28$, Correlación de Spearman). Asimismo, los resultados evidencian que el hecho de utilizar más o menos plantas combustibles del ambiente circundante no influye en una mayor predilección hacia la forestación dirigida a la construcción (Test Fisher Exact, $\mathrm{p}=0,429)$, el reparo $(\mathrm{p}=0,583)$, el uso leñatero $(\mathrm{p}=$ $0,609)$ y/o propósitos forrajeros $(\mathrm{p}=0,389)$. Los resultados indican que las motivaciones para la forestación son independientes del conocimiento ecológico tradicional sobre plantas leñosas al cual tienen acceso en su ambiente. Este saber, de carácter acumulativo a lo largo de la vida parece vincularse con una mayor aptitud en el cultivo en sí mismo que a un saber que amplifique nuevas visiones sobre la importancia de forestar. En cambio, las razones para forestar dependen de necesidades concretas y fundamentales de su vida cotidiana, vinculadas a su actividad pecuaria y a la necesidad de adaptarse a un clima adverso. Por ende, no parece ser un tipo de apreciación que varíe con la mayor experiencia ecológica tradicional, sin embargo es necesario tomar estos datos cautelosamente dado que se trata de una comunidad pequeña. En este sentido, en concordancia con Palm et al. (2009) y Carrol et al. (2011), la integración del conocimiento tradicional con nuevas prácticas de forestación parece contribuir positivamente en el mejoramiento de la calidad de vida de los pobladores.

El Escalamiento Multidimensional (MSD) mostró un arreglo global de datos adecuado (Figura 2; Stress $=0,17 \mathrm{y}$ $\left.\mathrm{R}^{2}=0,97\right)$. En coincidencia con lo dicho anteriormente, se distingue un cuerpo de puntos integrado y uniforme en relación de las respuestas vinculadas a las motivaciones para forestar que se separa espacialmente de la variable TEK que representa el conocimiento ecológico tradicional (Figura 2). Por otra parte, se observa que para los informantes, los criterios vinculados a forestar con fines de reparo y/o construcción son muy semejantes y se acercan en el espacio, mientras que las razones ligadas al uso del forraje y la leña son disímiles entre si y se alejan de las primeras. Este hecho habla de un gran acuerdo entre informantes sobre estas razones mostrando una alta consistencia y un sistema compartido de valoraciones.

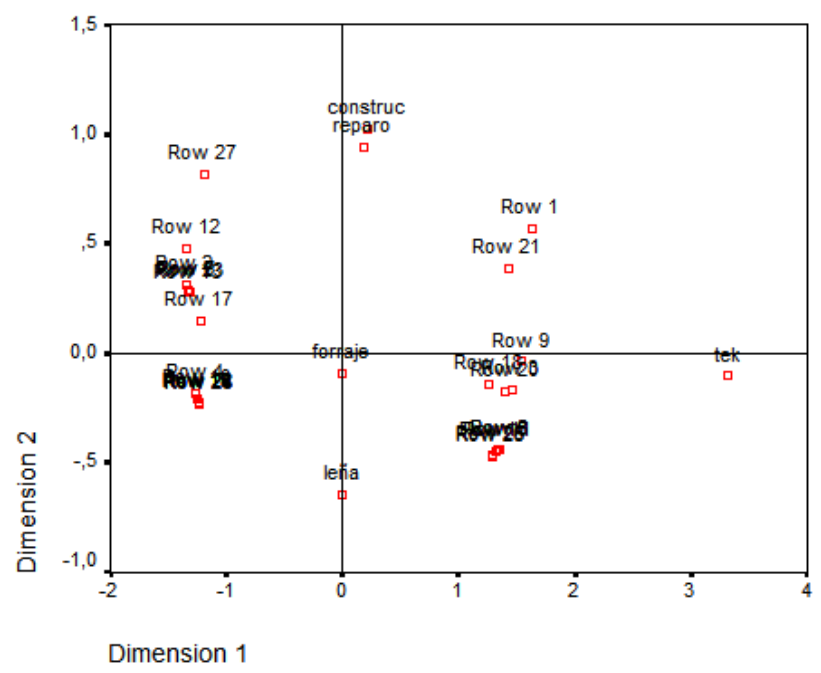

Figura 2. Escalamiento Multidimensional (MSD). Se representan los informantes (Row 1 al Row 28) y las respuestas vinculadas con las motivaciones para forestar: construcción (construc), reparo, forraje y leña. Asimismo, se representa la posición de la variable conocimiento ecológico tradicional (TEK).

\section{Conclusiones}

La forestación en el peri-domicilio es una estrategia multipropósito en las poblaciones rurales que tiene consecuencias a escala de paisaje, a nivel ambiental, social y familiar. Las motivaciones de los pobladores para efectuarla son variadas y complejas que conlleva la necesidad de estudios mayores, pero la idea de forestar en el peridomicilio con árboles exóticos para aprovisionarse de materiales de construcción y el reparo parece prioritario entre los pobladores.

Según nuestros datos estas decisiones son independientes del uso más o menos exhaustivo de la vegetación combustible nativa circundante, mostrando cierta disociación entre la práctica de recolección de leña (en la cual se prefieren los recursos nativos silvestres) y la práctica de forestación con exóticas (que para cerca de la mitad de los pobladores no tiene función leñatera primordial). Este hecho podría ser interpretado como que la forestación es una práctica generalista que está fuertemente influenciada a la transmisión oblicua llevada a cabo por 
técnicos externos de promoción agropecuaria que visitan y asisten a esta comunidad.

En concordancia con otros trabajos, este estudio muestra el rol de los agentes externos en la hibridación de nuevas prácticas ligadas con el ambiente (Eyssartier et al. 2011) y pone en relieve las posibles discrepancias entre las valoraciones de unos y otros.

También, resaltamos la necesidad de desarrollar proyectos para fomentar la práctica de forestación en los hogares y las escuelas rurales desde una perspectiva que tenga en cuenta los valores y prácticas de los pobladores locales. La forestación con especies nativas, a pesar de su lento crecimiento, aportaría otros significados y usos para los habitantes, restaurando un paisaje cultural que lentamente está siendo modificado por la intensificación del uso antrópico. Por estos motivos, es necesario seleccionar las especies adecuadas para la plantación, buscando un equilibrio ambiental y el mayor beneficio para los pobladores locales con especies de uso múltiple (Reubens et al. 2011).

\section{Agradecimientos}

Agradecemos profundamente a todos los pobladores de Pilkiniyeu del Limay quienes compartieron con nosotros su tiempo y conocimiento sobre su entorno vegetal. Este trabajo se realizó con el apoyo del CONICET (PIP 00337), la Agencia de Promoción Científica y Técnica de la Argentina (PICT 2007-02289) y la Universidad Nacional del Comahue.

\section{REFERENCIAS}

Agresti, A. 1996. An Introduction to Categorical Data Analysis. John Wiley \& Sons Inc., New York.

Albuquerque, U.P.; Lucena, R.F.P. \& Cunha, L.V.F.C. 2010. Métodos e Técnicas na Pesquisa Etnobiológica y Etnoecológica. Editora Livro Rápido/NUPEEA, Recife.

Alexiades, M.N. 1996. Selected Guidelines for Ethnobotanical Reserach: a field manual. The New York Botanical Garden, New York.

Alvarez, M.; Arach, A.; Arias, P.; Contreras, M.R.; Damau, I.B. \& Miño, G. 2009. Árboles de San Martín de los Andes. Guía de reconocimiento. Asociación Civil Pro-Patagonia, San Martín de los Andes.

Barreiro, G. 2007. Árboles de la Ciudad de Buenos Aires. Vázquez Mazzini Editores, Buenos Aires.

Berkes, F. \& Davidson-Hunt, I.J. 2006. Biodiversity, traditional management systems, and cultural landscapes: examples from the boreal forest of Canada. International Social Science Journal 187: 35-47.

Bran, D.; Ayesa, J. \& López, C. 2000. Regiones Ecológicas de Río Negro. Instituto Nacional de Tecnología Agropecuaria, San Carlos de Bariloche.

Broquen, P.; Falbo, G.; Apcarián, A.; Candan, F.; Girardin, J. \& Pellegrini, V. 2003. Relaciones entre las forestaciones, la erosión del suelo y la potencialidad productiva en la transición bosque-estepa (Andinopatagonia, Argentina). Investigación Agraria: sistemas y recursos forestales 12: 99-110.

Cabrera, A.L. \& Willink, A. 1973. Biogeografía de América Latina. Secretaría General de la Organización de los Estados Americanos, Programa Regional de Desarrollo Científico y Tecnológico, Washington, D.C.

Cardoso, M.B.; Ladio, H.A \& Lozada, M. 2010. Utilización de especies combustibles en una comunidad rural de la estepa patagónica. In: M.L. Pochettino, A.H. Ladio \& P. Arenas (eds), Traditions and Transformations in Ethnobotany. RISAPRET-CYTED, La Plata, p. 496-501.

Carrol, M.S.; Dhubháin, A.N. \& Flint, C.G. 2011. Back where they once belonged? Local response to afforestation in County Kerry, Ireland. Sociologia Ruralis 51(1): 35-53.

Correa, M.N. 1969-1998. Flora Patagónica (República Argentina). Colección Científica del Instituto Nacional de Tecnología Agropecuaria, Buenos Aires.
Davidson-Hunt, I.J. \& Berkes, F. 2003. Learning as you journey: Anishinaabe perception of social-ecological environments and adaptive learning. Conservation Ecology 8(1): 5. Disponible en http://www.conselcol.org/vol8/iss1/art5; acceso en 27 de junio 2011.

Eyssartier, C.; Ladio A.H. \& Lozada, M. 2011. Traditional horticultural knowledge change in a rural population of the Patagonian steppe. Journal of Arid Environments 75: 7886.

Ezcurra, C. \& Brion, C. 2005. Plantas del Nahuel Huapi: catálogo de la flora vascular del Parque Nacional Nahuel Huapi, Argentina. Universidad Nacional del Comahue, Red Latinoamericana de Botánica, San Carlos de Bariloche.

Hair, J.F; Anderson E.R.; Tatham R.L. \& Black, W.C. 1998. Multivariate Data Analysis. Prentice Hall, New Jersey.

ICEPH (Instituto Cordillerano de Estudios y Promoción Humana) 2010. Vivienda y Hábitat Campesino en la Patagonia. Ediciones ICEPH, San Carlos de Bariloche.

Izquierdo, F.; Velasco, V. \& Nasif, A. 2009. Montes Leñeros y Cortinas de Reparo en la Región Sur de Río Negro. Instituto Nacional de Tecnología Agropecuaria, San Carlos de Bariloche.

Jagger, P. \& Luckert, M.K. 2008. Investments and returns from cooperative and household managed woodlots in Zimbabwe: implications for rural afforestation policy. Land Use Policy 25: $139-152$.

Ladio, A.H. 2006. Uso y conservación de plantas silvestres con órganos subterráneos alimenticios en comunidades Mapuche de la estepa patagónica de la Argentina. In: U.P. Albuquerque \& C.F.C.B.R. Almeida (eds), Tópicos em Conservaçao e Etnobotânica de Plantas Alimentícias. NUPEEA, Recife, p. 53-72.

Ladio, A.H. 2011a. Underexploited wild plant foods of NorthWestern Patagonia. In: R. Filipi (ed.), Multidisciplinary Approaches on Food Science and Nutrition for the XXI Century. Transworld Research Network, Kerala, p. 1-16.

Ladio, A.H. 2011b. Traditional knowledge of edible wild native and exotic plants in the context of cultural change in human populations of arid Patagonia. Bioremediation, Biodiversity and Bioaviability 5(SI 1): 81-84.

Ladio, A.H. \& Lozada, M. 2009. Human ecology, ethnobotany and traditional practices in rural populations inhabiting the 
Monte Region: resiliencie and ecological knowledge. Journal of Arid Environments 73: 222-227.

Lebed, O.G. 2003. Cultivo de Plantas en la Estepa. PatagoniaArgentina. Ente para el desarrollo de la región y línea sur de la provincia de Río Negro, San Carlos de Bariloche.

Menni, A.M. 1999. A lo Largo de la Ruta 23. El imaginario popular y la comunicación humana, General Roca.

Molares, S. \& Ladio, A.H. 2009. Ethnobotanical review of the Mapuche medicinal flora: use patterns on a regional scale. Journal of Ethnopharmacology 122: 251-260.

Moles, A.T.; Gruber, M.A.M. \& Bonser, S.P. 2008. A new framework for predicting invasive plant species. Journal of Ecology 96: 13-17.

Nolan, J.M. 2001. Pursuing the fruits of knowledge: cognitive ethnobotany in Missouri's Little Dixie. Journal of Ethnobiology 21(2): 29-51.

Nuñez, M.A. \& Raffaele, E. 2007. Afforestation causes changes in post-fire regeneration in native shrubland communities of northwestern Patagonia, Argentina. Journal of Vegetation Science 18: 827-834.

Orellana, I.A. \& Raffaele, E. 2010. The spread of the exotic conifer Pseudotsuga menziesii in Austrocedrus chilensis forests and shrublands in northwestern Patagonia, Argentina. New Zealand Journal of Forestry Science 40: 199-209.

Palm, M.; Ostwald, M.; Berndes, G. \& Ravindranath, N.H. 2009. Application of Clean Development Mechanism to forest plantation projects and rural development in India. Applied Geography 29: 2-11.

Paruelo, J.M.; Golluscio, R.A.; Jobbágy, E.G.; Canevari, M. \& Aguiar, M.R. 2006. Situación ambiental en la estepa Patagónica. In: A. Brown, U. Martinez Ortiz, M. Acerbi \& J. Corchera (eds), La Situación Ambiental Argentina 2005. Fundación Vida Silvestre, Buenos Aires, p. 302-320.

Peralta C. 2002. Experiencias de Desarrollo Rural. Ediciones Instituto Nacional de Tecnología Agropecuaria, San Carlos de Bariloche.

Puntieri, J.G. \& Grosfeld, J.E. 2009. Arbolado urbano en la patagonia andina: buscando el equilibrio. In: Desde la Patagonia: difundiendo saberes. Vol. 9. Universidad Nacional del Comahue, San Carlos de Bariloche.
Radovich, J. \& Balazote, A. 1991. La represa de Piedra del Águila: la etnicidad Mapuche en un contexto de relocalización. América Indígena, Instituto Indigenista Interamericano, México.

Raffaele, E. 1996. Relationship between seed and spore banks and vegetation of a mountain flood meadow (mallín) in Patagonia, Argentina. Wetlands 16: 1-9.

Raffaele, E. 1999. Mallines: aspectos generales y problemas particulares. In: A.I. Malvárez (ed.), Tópicos sobre Humedales Subtropicales y Templados de Sudamérica. Universidad de Buenos Aires, Buenos Aires.

Raffaele, E. \& Veblen, T.T. 2001. Effects of cattle grazing on early post-fire regeneration of matorral in Northwest Patagonia, Argentina. Natural Areas Journal 21: 243-249.

Reubens, B.; Moeremans, C.; Poesen, J.; Nyssen, J.; Tewoldeberhan, S.; Franzel, S.; Deckers, J.; Orwa, C. \& Muys, B. 2011. Tree species selection for land rehabilitation in Ethiopia: from fragmented knowledge to an integrated multicriteria decision approach. Agroforestry Systems 82: 303330.

Rodríguez y López, S. 2006. Conocimiento y Utilización de Recursos Maderables en Comunidades Rurales de la Provincia de Córdoba, Argentina. Tesis de Licenciatura (Antropología Biológica y Cultural). Universidad Nacional de Córdoba.

Rusch, V.; Vila, A. \& Márques, B. 2008. Conservación de la Biodiversidad en Sistemas Productivos. Forestación del Noroeste de la Patagonia. Instituto Nacional de Tecnología Agropecuaria (INTA), San Carlos de Bariloche.

Sarasola, M.M.; Rusch, V.E.; Schlichter, T.M. \& Ghersa, C.M. 2006. Invasión de coníferas forestales en áreas de estepa y bosques de ciprés de la cordillera en la Región Andino Patagónica. Ecologia Austral 16: 143-156.

Steinbach, G.; Hofmann, R. \& Held, H. 2007. Árboles. Guías de Campo Blume. Blume, Barcelona.

Valdora, E.E. \& Soria, M.B. 1999. Árboles de Interés Forestal y Ornamental para el Noroeste Argentino. Laboratorio de Investigaciones Ecológicas de Las Yungas, Universidad Nacional de Tucumán, Tucumán. 